tracheobronchial tree during bronchoscopy, including normal bronchial epithelium, dysplastic mucosa and hilar lung cancer.

Methods The newly developed integrated-type ECS for the bronchoscope has a built-in two imaging system with a conventional mode and a high-power endocytoscopic mode. ECS has a high magnification of $570 \times$. Thirty-seven patients including 9 hilar lung cancer, 6 abnormal sputum cytology, 19 squamous dysplasia, and 3 after photodynamic therapy were entered into the study and underwent white light, narrow band imaging and autofluorescence imaging bronchoscopy. Both the abnormal area of interest and surrounding normal bronchial mucosa were stained with $0.5 \%$ methylene blue and examined with ECS. Histological examinations with haematoxylin and eosin stain were performed using the biopsied specimens. The ECS imaging was analysed and correlated with the corresponding histological examination.

Results ECS imaging could distinguish between different types of bronchial epithelium including normal bronchial mucosa, squamous dysplasia, and hilar lung cancer. Squamous dysplasia and hilar lung cancer were predictive with sensitivity of $85.7 \%(12 / 14)$ and $90.9 \%$ $(10 / 11)$ and specificity of $100 \%$ (12/12), respectively. These ECS images corresponded well conventional histology.

Conclusion ECS was useful for the discrimination between normal bronchial epithelial cells and dysplastic cells or malignant cells during bronchoscopy in real time. This novel technology has an excellent potential to provide in vivo diagnosis during bronchoscopic examinations.

\section{S37 COMPARISON OF DYNAMIC CONTRAST ENHANCED MRI (DCE-MRI) PARAMETERS WITH INTEGRATED PET-CT AND SERUM MESOTHELIN IN THE BASELINE ASSESSMENT OF MALIGNANT PLEURAL MESOTHELIOMA}

doi:10.1136/thx.2010.150912.37

${ }^{1} \mathrm{C}$ Hooper, ${ }^{2} \mathrm{D}$ Hall, ${ }^{3} \mathrm{P}$ Virgo, ${ }^{4} \mathrm{P}$ White, ${ }^{3} \mathrm{M}$ Darby, ${ }^{5} \mathrm{~T}$ Hall, ${ }^{6} \mathrm{~J}$ Braybrooke, ${ }^{2} \mathrm{~J}$ Searle, ${ }^{2}$ L Lyburn, ${ }^{1} \mathrm{~N}$ Maskell. ${ }^{1}$ Academic Respiratory Unit, Department of Clinical Sciences, University of Bristol, Southmead Hospital, Bristol, UK; ${ }^{2}$ Cobalt Cheltenham Imaging Centre, Cheltenham, UK; ${ }^{3}$ Southmead Hospital, North Bristol NHS Trust, Bristol, UK; ${ }^{4}$ University of the West of England, Bristol, UK; ${ }^{5}$ Royal United Hospital Bath, Bath, UK; ${ }^{6}$ Bristol Oncology Centre, UBHT, Bristol, UK

Integrated PET-CT scans and serum mesothelin measurement have shown early promise in predicting prognosis and evaluating treatment response in malignant pleural mesothelioma (MPM) but may be less reliable with sarcomatoid histology or prior talc pleurodesis. Dynamic Contrast Enhanced-MRI (DCE-MRI) with pharmacokinetic analysis is a novel metabolic imaging modality providing a measure of tumour blood flow and angiogenesis. We prospectively examined the relationship between pharmacokinetic parameters on DCE-MRI with PET-CT, serum mesothelin and histological subtype in MPM patients at diagnosis.

Method 30 pre-treatment patients with a histologically proven MPM underwent DCE-MRI and integrated PET-CT and serum mesothelin assay (MESOMARK) at a single visit. SUVmax and total glycolytic volume (TGV) were reported from PET-CT scans with TGV calculated using MIM software version 4.2.2 (MIMvista corp.). Gadolinium washout rate (GWR) on DCE-MRI was defined at a region of interest from a straight line fit to the kinetic curve data (CAD software-ViewForum R6.3 V1L3, Philips Medical Systems) between peak enhancement in the first $2 \mathrm{~min}$ and the last data point.

Results 70\% (21/30) epithelioid and 30\% (9/30) sarcomatoid histology. $43 \%(13 / 30)$ had undergone prior talc pleurodesis. Histology did not statistically significantly affect SUVmax, TGV or GWR. Serum mesothelin was significantly greater in the epithelioid group $(3.2 \mathrm{nM} / 1(2.0,6.3)$ vs $0.6 \mathrm{nM} / 1(0.5,0.8) \mathrm{p}<0.001)$. There was no significant difference in mesothelin, SUVmax, TGV or GWR between talc pleurodesed and non-pleurodesed patients in the whole group, but in the epithelioid sub-group there was a trend to significantly higher TGV with talc pleurodesis (talc: $2799(1931,11257)$ no talc: $955.5(146.8,2354) \mathrm{p}=0.053)$ that was not observed with GWR $(p=0.4179)$. While SUVmax strongly correlated to TGV $(r=0.725$, $\mathrm{p}<0.001$ ), there was no correlation between GWR and TGV $(r=0.203, p=0.282)$ or between mesothelin levels and any of the imaging values.

Conclusion Metabolic imaging has been proposed as an important component of the assessment and management of patients with malignant pleural mesothelioma. Gadolinium washout rate on DCE-MRI may be less sensitive to talc pleurodesis than PET-CT parameters and MRI is a cheaper, more readily available modality that involves shorter patient appointment times, warranting further study in MPM prognostic evaluation and treatment response monitoring.

\section{S38 FOURIER TRANSFORM INFRA-RED (FTIR) SPECTROSCOPY ON SPUTUM FROM LUNG CANCER PATIENTS, HEALTHY CONTROLS AND A HIGH-RISK COHORT}

doi:10.1136/thx.2010.150912.38

${ }^{1} \mathrm{R}$ Ghosal, ${ }^{1} \mathrm{~K}$ E Lewis, ${ }^{2} \mathrm{P}$ Kloer, ${ }^{1} \mathrm{~S}$ Bayliss, ${ }^{3} \mathrm{~L}$ Mur, ${ }^{1} \mathrm{P} \mathrm{D}$ Lewis. ${ }^{1}$ School of Medicine, Swansea University, Swansea, UK; ${ }^{2}$ Prince Philip Hospital, Llanelli, UK; ${ }^{3}$ Institute of Biological Sciences, Aberystwyth University, Aberystwyth, UK

Introduction New cheap and high throughput technologies may allow earlier diagnosis and cost-effective screening programmes for lung cancer (LC). We have shown that sputum is a feasible biofluid for FTIR spectroscopy analysis ${ }^{1}$ and now further evaluate FTIR in diagnosing LC.

Methods Sputum was taken from three groups: a) 54 patients (mean age $66.6 \pm 8.7$ years) with a histological diagnosis of LC (39 NSCLC, 9 small cell, 1 carcinoid, 5 clinical diagnosis). b) 24 patients (mean age 65.1 \pm 13.6 years) having bronchoscopy for possible LC

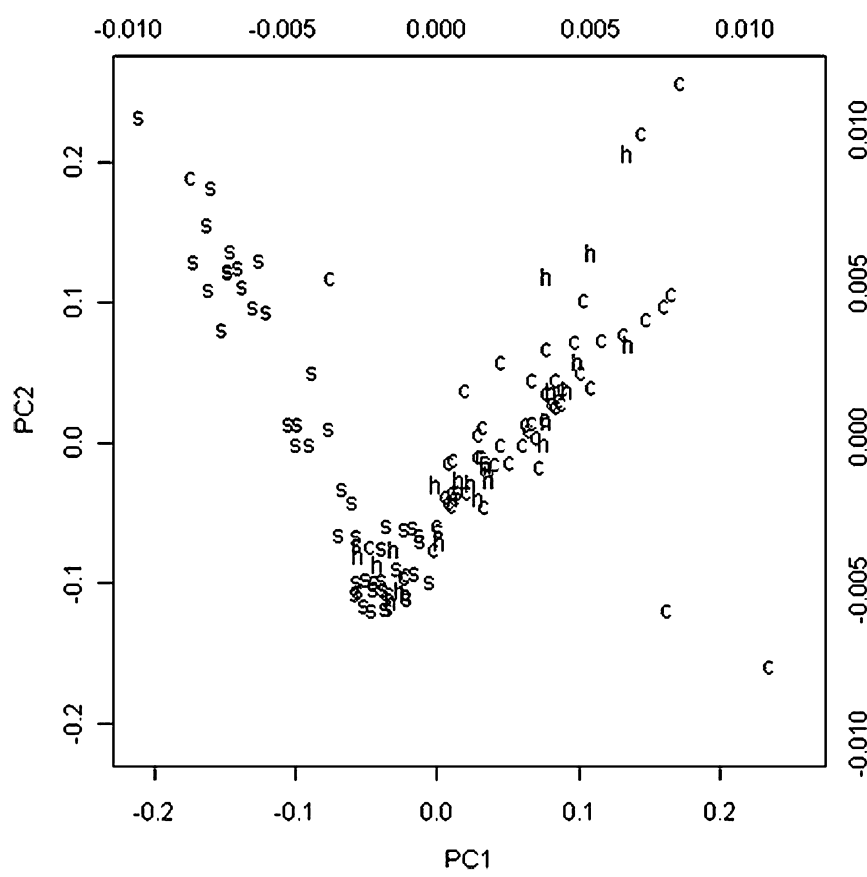

Abstract S38 Figure 1 Principal component analysis of cancer (c), healthy control (s) and high-risk (h) spectra. 
but no evidence of cancer was found after 1-year follow-up (highrisk). c) 54 healthy controls (HC) (mean age $51.1 \pm 15.3$ years) who had no history or symptoms of LC or known respiratory disease. Sputum was self-expectorated and frozen immediately at $-80^{\circ} \mathrm{C}$, thawed in batches, mucolytics were added then samples centrifuged at $3000 \mathrm{rpm}$ for $10 \mathrm{~min}$ to form pellets. FTIR was performed using the VERTEX 70 spectrometer (Bruker Optics Ltd, Banner Lane, Coventry, UK). Median absorbance values for each wavenumber for the LC and HC cohorts were compared, then principal component analysis (Abstract S38 Figure 1) and logistical regression identified the wavenumbers that provided the greatest accuracy in differentiating the two groups; the high-risk cohort was then applied to the predictive model to see if they could be correctly identified.

Results 126 light absorbance wavenumbers were significantly different between the LC and HC groups (each $p<0.05$ ). Two wavenumbers, $1031.7 \mathrm{~cm}^{-1}$ and $1409.7 \mathrm{~cm}^{-1}$ were used to develop a predictive model providing a sensitivity of $93 \%$ and specificity of $91 \%$. This model then predicted 17 of the 24 high-risk cohorts as LC.

Conclusion FTIR spectroscopy can distinguish LC from HC with high accuracy but had reduced specificity when applying high-risk patients, tending to over-diagnose LC. Follow-up will determine if these $17 / 24$ people are indeed false positives or have pre-cancerous molecular changes not identifiable by current methods.

\section{REFERENCE}

1. BTS Winter Meeting Abstract S19, Thorax 2007;62(Supplement iii):A10.
Abstract S39 Table 1

\begin{tabular}{|c|c|c|c|c|c|}
\hline Patients & HE & P63 & CK5/6 & TTF1 & Final diagnosis \\
\hline 1 & NSCLC-NOS & ND & ND & ND & NSCLC-NOS \\
\hline 2 & NSCLC-NOS & ND & ND & ND & NSCLC-NOS \\
\hline 3 & NSCLC-NOS & ND & ND & ND & NSCLC-NOS \\
\hline 4 & NSCLC-NOS & ND & ND & ND & NSCLC-NOS \\
\hline 5 & NSCLC-NOS & ND & ND & ND & NSCLC-NOS \\
\hline 6 & NSCLC-NOS? Squamous & ND & ND & ND & NSCLC-NOS? Squamous \\
\hline 7 & NSCLC-NOS? Adenocarcinoma & IT & IT & IT & NSCLC-NOS? Adenocarcinoma \\
\hline 8 & NSCLC-NOS & ++ & ++ & - & Squamous \\
\hline 9 & NSCLC-NOS & - & - & ++ & Adenocarcinoma \\
\hline 10 & NSCLC-NOS & - & - & ++ & Adenocarcinoma \\
\hline
\end{tabular}

$\mathrm{HE}$, Haematoxylin and Eosin; Squamous carcinoma markers - p63, cytokeratin 5/6 adenocarcinoma marker - Thyroid transcription factor 1 , ND, Not done, IT, Inadequate tissue.

Conclusion Thus we have shown that adequate tissue samples can be obtained at EBUS-TBNA and the frequency of NSCLC-NOS is less $(7 / 78=9 \%)$ compared to the histological bronchial biopsy samples. In cases, where morphological sub typing of NSCLC on HE is not possible, immunohistochemistry should be performed.

\section{S40 EARLY EXPERIENCE OF ENDOBRONCHIAL ULTRASOUND- MINIPROBE (EBUS-MP) FOR INVESTIGATION OF PERIPHERAL PULMONARY MASS LESIONS}

doi:10.1136/thx.2010.150912.40

J F Tiernan, W Wallace, K M Skwarski. Royal Infirmary of Edinburgh, Edinburgh, UK

Introduction Peripheral pulmonary mass lesions are common findings in respiratory medicine. The frequency of detection of such lesions is rising with increasing availability of radiological imaging techniques. Their aetiology may need to be established by tissue sampling to facilitate appropriate management, for example, suspected malignancy. Traditional investigations include CT-guided biopsy, bronchoscopic biopsy, endoscopic ultrasound with fine needle aspiration (EBUS/EUS) and surgical intervention. Each modality has potential complications, for example, pneumothorax following CT-guided biopsy. Endoscopic ultrasound miniprobe is established as a valuable tool, particularly in the staging of early GI tumours and in extraductal visualisation of the biliary tract. EBUSMP has been used for qualitative assessment of bronchial mural structures in lung transplant recipients but little is known about the role of EBUS-MP sampling of peripheral pulmonary mass lesions. The purpose of this paper is to demonstrate our experience with this technique to date.

Methods All EBUS-MP procedures were carried out over a 6-month period in a tertiary respiratory centre. Patients were referred for suspected malignancy. All procedures were undertaken by the same consultant bronchoscopist, assisted by a respiratory trainee. An Olympus UM-S20-17S $1.7 \mathrm{~mm}$ Miniprobe ${ }^{\circledR}$ was identify the target lesion. Samples (biopsies or endobronchial brushings) were then taken from the identified subsegmental bronchus. Each case was subsequently reviewed with respect to diagnostic rate, subsequent management, complications and potential alternative investigations to EBUS-MP.

Results 24 EBUS-MP procedures were performed on 22 patients (Age range 53-82 years (mean 70.4 years)). FEV1 ranged from $0.8 \mathrm{~L}$ to $2.9 \mathrm{~L}$. 20 of 22 CT-identified lesions $(14-60 \mathrm{~mm}$ ) were visualised with EBUS-MP. No complications occurred in study population. Abstract S40 Figure 1 shows detailed outcomes for EBUS-MP.

Conclusions EBUS-MP is a novel technique in bronchoscopy. Our early experience has demonstrated some potential usefulness of the procedure, allowing good visualisation of lesions. No complications have occurred to date. We believe that EBUS-MP sampling may have 